\title{
Sirt 6 as a novel target for neuroinflammation and brain aging
}

\author{
Lalita Subedi and Sun Yeou Kim* \\ Laboratoy of Pharmacognosy, College of Pharmacy and Gachon Institute of Pharmaceutical Sciences, Gachon University, Republic of Korea
}

World population is experiencing the complications of Alzheimer's disease (AD), the most common type of age related dementia. Sirtuins (Sirt1-7) are the nicotinamide adenine dinucleotide $\mathrm{NAD}^{+}$dependent deacetylases responsible for variety of human ailments like cancer, diabetes, inflammatory disorders and neurodegenerative disorders. Sirt1 is associated with apoptosis, differentiation, aging and oncogenic transformation where Sirt 2 act as a mitotic checkpoint protein. Sirt 3 control the superoxide level, and Sirt 4 controls the fatty acids oxidation and mitochondrial gene expression. Sirt5 expression is responsible for metabolic changes during aging. Sirt6 and Sirt7 are responsible for histone acetylation in aging brain [1]. Shift in homeostatic balance of inflammatory mediators to proinflammatory state is the characteristic feature during brain aging. Hence correlation between proteins related with brain aging with neuroinflammation is an obvious phenomenon [2]. Normal brain aging is responsible for late-life diseases such as neuroplasticity, depression, psychiatric, neurological and neuroinflammatory disorder etc. via changes in gene function over time [3]. Studies reported that Sirt1 is associated in neuronal development, synaptic plasticity and memory formation via increasing the axonogenesis, neurite outgrowth, and dendritic arborization where Sirt2 have the opposite function, whereas little is known about Sirt3-7 in the brain. Sirt6 protein levels decline during neuroinflammation and brain aging although the exact mechanism is unknown [1]. Sirt6 regulates various genes associated in stress response and aging, hence it showed promising role in controlling the aging like degenerative processes [4]. Expression of Sirt6 decreased in various inflammatory conditions like ischemia, stroke and other disease but the exact role of Sirt6 in brain function and neuronal survival is still unknown [5]. Sirt6 inhibits NF- $\mathrm{BB}$ dependent transcription by affecting chromatin structure and also regulates the cardiac hypertrophy and various other inflammatory ailments. Sirt1 and Sirt6, regulates NF- $\mathrm{kB}$ activity via decreasing promoter accessibility to $\mathrm{p} 65$. Components of innate immunity like macrophages, cytokines, complement, and pattern-recognition receptors etc. play a key role in CNS homeostasis characterized by neuroinflammation. Sirt6 mediated positive control of proinflammatory cytokine as well as NF$\kappa \mathrm{B}$ might play a major role in controlling the neuroinflammation [6]. A strong evidence of the function of Sirt6 in the crosstalk between the ageing, metabolic syndrome and osteoarthritis was reported already [7]. Its anti-inflammatory potential in human umbilical vein endothelial cells was also reported recently. Genetic knockdown of Sirt6 significantly increase the production of inflammatory mediators like IL-6, IL-8, COX-2, IL-1 $\beta$, PGE $_{2}$, PGF $_{2 \alpha}$, MMP-2, PAI-1, MMP9; angiogenic protein like VEGF and FGF2 together with increase in cell migration during endothelial cell dysfunction induced by LPS [8]. Sirt6 is essential for sodium sulfide-mediated cytoprotective effect in ischemia/reperfusion-stimulated brain endothelial cells [9]. Sirt6 downregulates the transcription of hypoxia-inducible factor (HIF1 $\alpha$ ) target genes during tumorigenesis and inflammation. Furthermore, NF-kB inhibitory activity of Sirt6 can control the inflammation and aging $[10,11]$. The exact role and molecular mechanism of Sirt6 in neuroinflammation and neurodegenerative disease has not been studied yet, however, the role of Sirt6 in downregulating inflammatory mediators, proinflammatory cytokines, transcription factors like NF$\mathrm{kB}, \mathrm{HIF}$ and neuroinflammation inducing signaling pathways provides a key for its activity to control neuroinflammation, aging and related dementia such as AD.

Sirt6 controls the inflammatory mediators of various types of inflammatory and aging related complications suggesting that Sirt6 may play a pivotal role in controlling the neuroinflammatory disorders as well. Moreover sirt6 is compulsory for the life survival, slow aging and treatment of inflammation and cancers where critical stages of inflammation fall. Taken together, sirt6 is key mediator in the regulation of various neuroinflammatory conditions like Alzheimer disease, Parkinson's disease, amyotrophic lateral sclerosis, ischemia, stroke, multiple scleroses, brain aging etc.

\section{References}

1. Braidy N, Poljak A, Grant R, Jayasena T, Mansour H, et al. (2015) Differential expression of sirtuins in the aging rat brain. Front Cell Neurosci 9: 167. [Crossref]

2. Sparkman NL, Johnson RW (2008) Neuroinflammation associated with aging sensitizes the brain to the effects of infection or stress. Neuroimmunomodulation 15: 323-330. [Crossref]

3. Sibille E (2013) Molecular aging of the brain, neuroplasticity, and vulnerability to depression and other brain-related disorders. Dialogues Clin Neurosci 15: 53-65. [Crossref]

4. López-Otín C, Blasco MA, Partridge L, Serrano M, Kroemer G (2013) The hallmarks of aging. Cell 153: 1194-1217. [Crossref]

5. Cardinale A, de Stefano MC, Mollinari C, Racaniello M, Garaci E, et al. (2015) Biochemical characterization of sirtuin 6 in the brain and its involvement in oxidative stress response. Neurochem Res 40: 59-69. [Crossref]

6. Lehnardt S (2010) Innate immunity and neuroinflammation in the CNS: the role of microglia in Toll-like receptor-mediated neuronal injury. Glia 58: 253-263. [Crossref]

7. Ailixiding M, Aibibula Z, Iwata M, Piao J, Hara Y, et al. (2015) Pivotal role of Sirt6 in the crosstalk among ageing, metabolic syndrome and osteoarthritis. Biochem Biophys Res Commun 466: 319-326. [Crossref]

Correspondence to: Sun Yeou Kim, Laboratoy of Pharmacognosy, College of Pharmacy and Gachon Institute of Pharmaceutical Sciences, Gachon University, Incheon, 406-799, Republic of Korea, Tel: +82-32-899-6411, Fax: +82-32-8998962, E-mail: sunnykim@gachon.ac.kr

Received: August 10, 2016; Accepted: August 20, 2016; Published: August 22, 2016 
8. Lappas M (2012) Anti-inflammatory properties of sirtuin 6 in human umbilical vein endothelial cells. Mediators Inflamm 2012: 597514. [Crossref]

9. Hu Y, Li R, Yang H, Luo H, Chen Z (2015) Sirtuin 6 is essential for sodium sulfidemediated cytoprotective effect in ischemia/reperfusion-stimulated brain endothelial cells. J Stroke Cerebrovasc Dis 24: 601-609. [Crossref]
10. Kawahara TL, Michishita E, Adler AS, Damian M, Berber E, et al. (2009) SIRT6 links histone $\mathrm{H} 3$ lysine 9 deacetylation to NF-kappaB-dependent gene expression and organismal life span. Cell 136: 62-74. [Crossref]

11. Zhang N, Li Z, Mu W, Li L, Liang Y, et al. (2016) Calorie restriction-induced SIRT6 activation delays aging by suppressing NF-kappaB signaling. Cell cycle 15: 1009-1018. [Crossref]

Copyright: $(02016$ Subedi L. This is an open-access article distributed under the terms of the Creative Commons Attribution License, which permits unrestricted use, distribution, and reproduction in any medium, provided the original author and source are credited. 